\title{
Consumers' level of comfort with an advanced practice role for registered nurses in general practice: A Queensland, Australia study.
}

Desley Hegney RN, DNE, BA (Hons), PhD

Director, Research and Practice Development Centre, University of Queensland and Blue Care and Professor, Faculty of Nursing, University of Queensland

Robert Eley, BSc (Hons), MSc, PhD, CIBiol, Senior Research Fellow Centre for Rural and Remote Area Health, University of Southern Queensland

Elizabeth Buikstra, BSc (Hons), PhD, Research Fellow

Centre for Rural and Remote Area Health, University of Southern Queensland

Sharon Rees RN, Project Officer

Centre for Rural and Remote Area Health, University of Southern Queensland

Elizabeth Patterson RN, BSc, MHSc (Nurs), PhD

Associate Professor and Dean

School of Nursing and Midwifery, Griffith University - Gold Coast Campus,

PMB 50, Gold Coast 9726

Corresponding author's address:

Dr Rob Eley

Centre for Rural and Remote Area Health

University of Southern Queensland

PO Darling Heights

Toowoomba Qld 4350

AUSTRALIA

Email: eleyr@usq.edu.au

Phone: +61 746315457 


\begin{abstract}
A study was carried out in Queensland, Australia which aimed to ascertain how comfortable consumers would be with an advanced practice role for nurses employed in general practice. Metropolitan, rural and remote areas were represented. The self-report questionnaire was completed by 87 females and 19 males. Respondents were most comfortable with nurses giving vaccinations and managing treatment of wounds. They were least comfortable with nurses diagnosing and treating minor illnesses. No differences in responses to the level of comfort in nurses performing certain tasks were detected due to age or sex. Residence in remote locations affected responses to two of the 25 offered tasks. Results demonstrated that the majority of consumers are either very comfortable or comfortable with a wide range of services that nurses could provide. These results may be used to expand the role of practice nurses to complement the services provided by general practitioners.
\end{abstract}

Keywords: Practice nurses, general practice; questionnaire, consumer perceptions Word Count:

\title{
Introduction
}

As reported by the Productivity Commission (2005), there is a workforce shortage across many health professions in Australia, resulting in a need for reform and new models of care. The Australian Government seeks to identify initiatives that would produce a more sustainable and responsive health workforce, while maintaining a commitment to high quality and safe health outcomes (Productivity Commission, 2005).

Advancing the nurse's role in general practice is one strategy that has been implemented. As part of the 2001-2002 federal budget, the Practice Incentives 
Program was introduced providing support for general practitioners (GPS) in rural and other areas of need to employ nurses. Further funding was allocated to metropolitan GPs in the 2002-2003 budget (Australian Government Department of Health and Ageing, 2003). This practical incentive to employ practice nurses (PNs) and Aboriginal Health Workers in general practice has been followed by new Medicare Benefits Schedule (MBS) item numbers for wound dressings, immunisations and Pap smears provided by a PN.

Various studies have investigated practice nurses' perceptions of their role. In the main, these studies suggest that PNs perceive their role as being assistive and therefore practise accordingly (Bonawit \& Watson, 1996; Dunt, Temple-Smith, \& Johnson, 1991; Keyzer, Hall, Mahnken, \& Keyzer, 1996; Patterson, Del Mar, \& Najman, 1999a; Patterson, Del Mar, \&. Najman, 1999b). However, a more recent study conducted throughout Australia, and funded by the Department of Health and Ageing, concluded that the future PN will undertake a greater integration role with more time spent on clinical care and clinical organisation, and less time spent on practice administration (Royal Australian College of General Practitioners \& Royal College of Nursing Australia, 2004).

Factors identified as limiting the development of the $\mathrm{PN}$ role include a lack of funding to employ them and to provide for continuing education opportunities, 'gate keeping' of special interest groups, and general practitioners reluctance to relinquish any 'medical work' to nurses (Condon, Willis, \& Litt, 2000; Le Sueur \& Barnard, 1993; Patterson et al., 1999a; Patterson et al., 1999b). Interestingly, one of the most striking differences between two countries where practice nurses are commonly used was the attitude of the medical profession - relatively hostile to the extended role of nurses in the US, and supportive in the UK (Buchan \& Calman, 2005) 
A recent review notes that most of the evidence of the use of nurses in 'advanced practice roles' demonstrates their clinical effectiveness and cost effectiveness (Buchan \& Calman, 2005). The review recognises that comparison across countries is made difficult by different definitions and roles. However in primary care settings the data overwhelmingly supports the notion that nurses can provide care which is equivalent to that provided by doctors in these settings. In fact patients were generally more satisfied with nurse consultations than with doctor consultations.

Although GPs and PNs have been the target of surveys it is somewhat surprising that consumers' perceptions of the role of PNs and their acceptance of nurses undertaking responsibilities previously undertaken by GPs received little attention in Australia until 2003. In that year two studies were undertaken. One study was undertaken for submission to The National Steering Committee on Nursing in General Practice (Cheek et al., 2002). Data were collected through the use of 20 focus groups in six states and territories and a thematic analysis undertaken. Participants in the study, including carers, the elderly, and those who identified that they had a chronic disease had varying levels of contact with general practice. The authors found that generally consumers had a lack of awareness of the actual or potential scope of nursing in general practice. However, those consumers with a greater exposure to PNs, (such as carers and persons with a chronic illness) were able to envisage roles for nurses that extended beyond the traditional role identified in previous Australian surveys conducted by Patterson et al (1999a; 1999b).

The identified expanded role included development of care plans, providing education and support, prescribing continuing medications and undertaking Pap Smears. However, consumers also expressed concerns about expansion of the role. For example, they were concerned about medico-legal problems for the doctor, possible substitution of doctors by PNs and increased costs to the consumer for PN 
visits. In particular, consumers stated that their choices should remain and neither nurses nor GPs should act as gatekeepers to each other (Hegney, Price, Patterson, Martin-McDonald, \& Rees, 2004). Overall, however, consumers were accepting of nurses in general practice because they trusted the GPs to employ suitably qualified and competent nurses. They expected that GPs and PNs would work collaboratively and in the best interests of the consumer (Cheek et al., 2002).

The study reported herein was undertaken at the same time as that of Cheek et al. (2002). The project was undertaken in Queensland and with the use of a self report questionnaire aimed to investigate how comfortable consumers would be with an extended nurse role within general practice. Some qualitative results have already been published (Hegney et al., 2004; Patterson, Price, \& Hegney, 2005). The authors describe similar concerns as those found by Cheek et al. with the right to see their health practitioner of choice maintained. Consumers, especially those in rural areas, believed that PNs should enhance, not replace, the GP. The expressed benefits of PNs included more time being made available to GPs and a more holistic and family oriented approach offered by the nurses than that offered by GPs.

An essential part of the data collection tools in the Queensland study was the use of a comfort scale developed specifically for this study. Results from that methodology are presented herein.

\section{Methodology}

\section{Participants}

All community members who were consumers of general practice care were eligible to participate in the study. The study aimed to include people who (a) were of either male or female gender; (b) were from English, Indigenous, or English as second language backgrounds; (c) accessed different types of general practice; (d) 
frequently, sometimes, or rarely visited a general practice; (e) attended practices where PNs had been employed for at least 12 months; and (f) attended practices where there were no PNs. To ensure that people who were most likely to access general practice were included, the following groups were targeted: (a) parents or carers of young children; (b) people with a chronic disease; and (c) older people (defined as over 70 years of age).

Participants were recruited from remote, rural and metropolitan areas as classified by the Rural, Remote and Metropolitan Area Classification 1991 Census Edition (Department of Primary Industries and Energy and Department of Health and Human Services, 1994). In rural and outer metropolitan areas recruitment was through individual general practices and through a Division of General Practice both of which distributed brochures and consent forms to potential participants. In other areas recruitment was through community groups (e.g., Indigenous health service, sporting/religious group, mental health service), and other contacts from within the community.

Information was collected by questionnaire from 106 participants prior to interviews held with one or two individuals (10 interviews) or in small focus groups of three or more people (17 focus groups).

\section{Materials}

The questionnaire was developed from a number of sources including a literature review and fact sheets from the Australian Government Department of Health and Ageing (ref). The questionnaire comprised items to elicit demographic data (e.g., gender, age and geographical location), frequency and reasons for attending a GP, practice data (e.g., number and gender of doctors and practice nurses) and level of comfort with potential PN activities. 
There were a total of 14 questions. Ten were categorical items requiring circling one of several possible answers (e.g. male or female) and one required entering a postcode. There were two further categorical items (questions 11 and 13). Question 11 on reasons for attending a doctor allowed for multiple responses to the 10 offered choices and a free text. Question 13 asked for a ranking of preference for seven means of communication about changes taking place in the doctor's surgery.

The final question (Question 14) listed 25 services that an appropriately trained and qualified PN could provide. Participants were requested to indicate on a five-point Likert scale $(1=$ very comfortable, 2 = somewhat comfortable, $3=$ undecided, $4=$ somewhat uncomfortable and $5=$ very uncomfortable) how comfortable they would be with a PN providing each service.

Prior to use in the main study, the questionnaire was piloted by three nurses, one general practitioner and four consumers for readability and understanding. Their suggestions were incorporated into the questionnaire.

\section{Procedure}

A plain language statement and consent form was developed in brochure format. The brochure described the study and provided contact details for the research team at the University of Southern Queensland (USQ). The brochure was provided to potential participants who were then asked to sign and return them to their general practice, post them to USQ or hand them personally to the research team members. Questionnaires were given to the 106 participants prior to individual interview or focus group. The research team provided clarification of any items if requested but ensured the privacy of each participant when they were completing the questionnaire. The questionnaire took approximately 15 minutes to complete. 
Interviews and focus groups took up to one and two hours, respectively. Questions and prompts developed for this process acted as a guide. Resulting discussions were allowed to follow their natural course allowing participants and researchers to explore ideas as they arose.

\section{Statistical Analyses}

Analyses conducted on the sample quantitative data were performed using the Statistical Package for Social Scientists (SPSS) program, version 11.5. Descriptive statistics, such as percentages, means and standard deviations, related to each survey question were calculated. Chi-squared analysis was undertaken to determine if differences existed between age or place of residence with sex, frequency of visits to a GP and contact with a PN. Analysis of variance was used to determine differences in the level of comfort of respondents of PNs performing certain tasks and age, location of residence and frequency of contact with GPs. Post hoc tests were undertaken using Least Squares Difference tests with Bonferonni adjustement.

\section{Ethics approval}

Ethics approval for this study was obtained from the University of Southern Queensland's Human Research and Ethics Committee.

\section{Results}

\section{Demographic data}

Of the 106 participants, $18 \%(N=19)$ were male, and $82 \%(N=87)$ were female. Twelve participants were Indigenous, 25 had young children, 37 had chronic disease and 5 came from a non-English speaking background. 
Table 1 outlines the number and percentage of participants in each age group. As only two participants were less than 20 years of age and only five 80 years of age or older these data were combined with the 20 to 29 and 70 to 79 age groups, respectively. Insert table 1 here

There were no differences in age owing to $\operatorname{sex}\left(\chi^{2}(5)=7.65, p>.05\right)$, how often they had seen a GP $\left(\chi^{2}(10)=12.50, p>.05\right)$, whether the GP's surgery had a nurse employed $\left(\chi^{2}(10)=18.28, p>.05\right)$, the length of time the surgery had a nurse employed $\left(\chi^{2}(5)=8.71, p>.05\right)$ and whether the respondents had any contact with a practice nurse $\left(\chi^{2}(5)=5.66, p>.05\right)$.

One hundred of the participants lived in five main locations in Queensland. The number, percentages and ages of participants in each of these locations are outlined in Table 2. There were significantly fewer participants under 50 in the two remote locations of Longreach and Roma than in the other three locations $\left(\chi^{2}(4)=\right.$ 19.32, $p<.05)$. Insert Table 2 here

There were no differences in place of residence by $\operatorname{sex}\left(\chi^{2}(4)=8.13, p>.05\right)$, how often they had seen GP $\left(\chi^{2}(8)=13.17, p>.05\right)$, whether the GP's surgery had a nurse employed $\left(\chi^{2}(8)=8.65, p>.05\right)$, the length of time the surgery had a nurse employed $\left(\chi^{2}(8)=11.16, p>.05\right)$ and whether patients had contact with a practice nurse $\left(\chi^{2}(4)=1.61, p>.05\right)$.

Most of the participants $(83,78.3 \%)$ indicated that the surgery they attended had a practice nurse. Only $6(5.7 \%)$ reported that the practise had no practice nurse and $17(16.0 \%)$ were not sure. Of the 83 who responded yes 65 (81.3\%) indicated that the GP surgery had employed a practice nurse for more than 12 months. The 
majority of respondents to the study (72\%) had had contact with a practice nurse; however most $(80 \%)$ were unaware of qualifications.

The major reasons for attending a GP are given in Table 3 with script renewal and health assessment or screening stated as reasons by more than $50 \%$ of the respondents. Over $50 \%$ of the women went to the GP for Pap smears and 25 of the 106 respondents for immunisation. Insert table 3 here

In response to the question as to preference for information regarding changes at their GP's surgery, the majority of respondents (62.4\%) wanted to be advised by letter. The second preference (33\%) was through a newsletter. The least preferred options for advice were television and radio (2.2\% each).

Comfort of participants to the role of the Practice Nurse

The major focus of the questionnaire was to ascertain the participants' levels of comfort with the advanced role of the practice nurse. Table 4 provides the number of responses, means and standard deviations for each of the items in the subscale that measures consumer's level of comfort with practice nurses performing the designated services. Insert Table 4 here.

Participants were most comfortable (over $70 \%$ of participants very comfortable) with practice nurses giving vaccinations and managing the treatment of wounds followed by providing education related to health issues, performing home safety checks for falls risks and doing baby checks. The areas that participants were less comfortable (fewer than $40 \%$ of participants very comfortable) with were: diagnosis and treatment of minor illnesses, prescribing legally sanctioned medication following medical guidelines and performing medical assessments for insurance. 
Fewer than $20 \%$ of participants were very uncomfortable with practice nurses performing any of the tasks that were offered.

There were no statistical differences $(p>.05)$ for any of the 25 items with age or number of times that a patient had contact with their GP. There were however statistically significant effects between the location of residence and the level of comfort with practice nurses giving test results, $(F(4,87) 5.59, p<.05)$. Significant differences were found between Longreach and Toowoomba $(t=-1.61, p<.05)$, Longreach and Gold Coast $(t=-1.39, p<.05)$, Longreach and Brisbane $(t=-1.38, p$ $<.05)$ and Longreach and Roma $(t=-1.30, p<.05)$. In all cases participants from Longreach were less comfortable with practice nurses giving test results than were participants from other locations.

Significant effects were also found between location and the level of comfort with practice nurses providing counselling or grief counselling $(F(4,84) 4.02, \mathrm{p}<$ .05). Roma differed from Toowoomba $(t=-1.16, p<.05)$, Brisbane $(t=-95, p<.05)$ and Gold Coast $(t=-93, p<.05)$. In all cases participants from Roma were less comfortable with PN providing counselling than those participants from Toowoomba, Brisbane and Gold Coast.

\section{Discussion}

The results of this study determined consumers' perceptions and level of comfort with PNs performing advanced nursing practice roles in general practice. Participants were recruited through contacts and this may mean that the results are biased towards those people who are members of community groups and those who had time to be interviewed or attend focus groups. However as many of the results concur with a national study undertaken at the same time (Cheek et al 2002) there is confidence that the results do reflect consumer perceptions and preferences. 
An additional limitation to the study was that the word comfortable was not defined to the participants. Interpretation by individual participants of the question "how comfortable would you be with a practice nurse performing [certain services]?" is not known. The assumption by the researchers in designing the questionnaire was that comfort would be indicative of a mental state of being at ease rather than a physical state of pain or discomfort.

Walker (2006) cites a recent unpublished survey by the Australian Practice Nurses Association that there are between 5500 and 6500 PNs in Australia with $50 \%$ of practices of 4-8 GPs employing nurses. In this study $77.4 \%$ of the respondents attended surgeries with two or more GPs and $78.3 \%$ of the total respondents indicated that the practice employed PNs.

Most respondents in the study did not know the qualifications of the nurses at their practice. Yet, despite this, most participants in this study were comfortable, if not very comfortable with PNs expanding their role in general practice. These findings concur with Cheek et al. (2002) who found that consumers were generally accepting of nurses in general practice. They explained that consumers accepted this new role because they trusted the doctor to employ suitably qualified and competent nurses and they expected that GPs and PNs would work collaboratively and in the best interests of the consumer.

The level of comfort in nurses performing a wide variety of tasks was highest for vaccinations and management of wounds. These are two tasks for which MBS rebates are available when undertaken by PNs and it is possible that respondents had experience of this. The third idem on the MBS rebate scheme - Pap smears - 
was rated as one of the lowest in relation to level of comfort. At the time of the survey this procedure did not qualify for rebate.

With only two exceptions, level of comfort was not affected by sex, age or location of residence. In both exceptions it was a remote location that differed from the others. It has been noted that concern about PNs being substitutes for the GP is most evident in rural and remote areas ((Hegney et al., 2004). The results indicating that remote area respondents were least comfortable with nurses giving test results or counselling could be a reflection of this.

The qualitative data from the study (Hegney et al., 2004) indicate that, in agreement with the national survey (Cheek et al., 2002), participants were not really aware of the role of PNs and referred instead to their experience with nurses in an acute setting. In the Cheek et al study this lack of understanding, and a higher proportion of participants who had no contact with a PN, meant that the consumer was unable to visualise expansion of the role. Despite the lack of understanding and perhaps because of the higher degree of prior exposure to PNs in this study in Queensland it appears that participants could visualise expansion to the additional tasks that were offered.

In conclusion, the results of this study largely concur with those of the national study undertaken at the same time. However, they do offer a different perspective in that quantifiable data were collected. Recognition that GPs, PNS and consumers are all key stakeholders to improvement in health service provision appears to have been somewhat lacking in development of initiatives. The cumulative data from the study reported herein, the accompanying qualitative data (Hegney et al., 2004) and those from a national study (Cheek et al., 2002) now provide policy makers and practitioners alike with the necessary information as to what consumers want. 